\title{
Theory of Mind Abilities and Social Competence in Preschool Children
}

\author{
Shrunga Manchanapura Shivalingaiah ${ }^{1}$ Fathimath Ramseena ${ }^{1} \quad$ Nafeesath Shareen ${ }^{1}$ \\ ${ }^{1}$ Nitte Institute of Speech and Hearing, Mangalore, Karnataka, India \\ Address for correspondence Shrunga Manchanapura Shivalingaiah, \\ MASLP, Nitte Institute of Speech and Hearing, Mangalore, Karnataka \\ 575018, India (e-mail: msshrunga@gmail.com).
}

J Health Allied Sci ${ }^{\mathrm{NU}} 2019 ; 9: 9-11$

\begin{abstract}
Background and Objective Theory of mind (ToM) is the ability that can be attributed to mental status beliefs, intents, emotions, proficiency, etc., to oneself and to others, and the understanding that others also have intentions, conceptions, desires, and perspectives that are different from one's own. Daily social life depends on the ability to evaluate the behavior of other people on the basis of their mental state such as their beliefs, intentions, compassions, and goals. This study was conducted to explore the ToM abilities in preschoolers.

Methods A total of 36 preschoolers participated in the study. Two stories were narrated to the children, the classic Sally-Anne Task and the Smarty's Task. In both the tasks, the responses of the children were scored as either true belief or false belief. Results The results of the present study revealed mixed responses among the preschoolers. It was found that 3- to 4-year-old children had more false beliefs for

Keywords

- theory of mind

- preschoolers

- Sally-Anne task

- Smarty's task

both the tasks when compared with 4- to 5-year-old.

Conclusion This study highlights the importance of ToM abilities in typically growing children and other clinical population. It can be concluded that the ToM abilities were improved in typically developing children. Future studies are required to explore the higher levels of embedding of ToM, and also to incorporate it in the clinical population.
\end{abstract}

\section{Introduction}

Daily social life depends on the ability to evaluate the behavior of other people on the basis of their mental state such as their goals, emotions, intentions, and beliefs. This is accomplished by dedicated cognitive systems, collectively referred to as theory of mind (ToM). ToM gives an evolutionary advantage of reducing our susceptibility to deception. In brief, having a ToM is to be able to reflect on the contents of one's own and other's minds. In social situations, people with good ToM skills outsmart those with poor ToM. ToM is the ability to attribute to mental status beliefs, covet, conception, intents, emotions, proficiency, etc., to oneself and to others, and understanding that others also have intentions, conceptions, desires, and perspectives that are different from one's own. The term 'theory of mind' is the ability to represent the mental state to the self and to others. ${ }^{1}$ A task involving the action of a mistaken belief, that is, the false belief task, ${ }^{2,3}$ which became one of the most used tests for accepting a child with a ToM. The two narrow definitions on this task were (1) the research mainly focused on children aged 3 to 5 years because it is the period in which children start to achieve success in false-belief tasks and (2) prominence on relating mental conditions of the assumption, knowledge, and also emotions, purposes, desires, impulsion, thoughtfulness, understanding, and so on. However, Theory of Mind is one and only intimation that develops throughout the life time. Misconception of ability to understand the connection with different areas related to the society which involves the detection of mistakes one has done, communication, and simulation. ${ }^{4-7}$ This implies that falsebelief conception is referred to the ability of young children to make suitable or adjust to their universe of discourse.

Simultaneously, the two different opinions collide between 3-year-old children, that is, lack of success on 
misbelieve tasks and also noticeable achievements in transacting social interactions in everyday life. These bring us to the question whether the ToM has any indication or relevance in a child's ability to social behavior. ${ }^{8}$ Therefore, researchers give a wide statement on ToM which encloses a broad array of mental conditions from the attribution of intention, perception of attitude, emotion, intention, and cognition. During preschool age, most of the children grasp the mental abilities, mainly emotions. In particular, preschoolers can recognize and understand other people and also some amount of their thoughts and emotions: (1) they are not actually aware of what comes into sight, (2) they will exhibit some action or behavioral responses to some occasions which affect their current mood, (3) they can have two disputing emotions to a certain extent which happen at the same time. ${ }^{9}$ This will make the children understand people's emotions and these developments make the children much more skilled "mind readers." This will help the children to develop and change or convert their social interactions. According to this view, the preschooler's emotional understanding will be significantly associated with the companion groups feelings, understandings, sympathy and to accept the usage of socially conducted acts too versa the emotional expressions. As mentioned before, these outcomes in mind reading not only allow the interconnection between social events and social gathering, but they also look for the people who are engaging in the social interactions with these children.

Till date, there is a dearth of available data on ToM up to the third level for children, especially in India. Also, it will be interesting to study the relation between the social competence of the child and the emergence of different levels of ToM.

\section{Objectives}

The main objectives of the study were to understand the developmental pattern of ToM and the relation between ToM abilities and the social competence of the child.

\section{Methods}

A total of 36 preschoolers between age 3 and 5 years (preschoolers) with normal speech and language development participated in this study. The study was conducted after obtaining consent from the school authority and parents.

A set of stories were used for testing ToM abilities. Two stories were narrated to the children-the classic Sally-Anne Task and Smarty's Task. Children were tested individually in a quiet room within the school premises. Two tests were performed in one session that took approximately 20 minutes. The experimenter took a little time for warm up by asking questions and playing with the children. After each story, the participants were asked a series of forcedchoice questions about information in the preceding story. Verbal positive reinforcement was given to the children for all tasks, regardless of correctness.

\section{Task 1 (Sally-Anne Task) ${ }^{3}$}

The child was made to sit facing the experimenter and two dolls were introduced to the child named as Sally and Anne. Sally had a basket and Anne had a box in this play. A story was narrated to the child as: "Sally and Anne are playing with the marbles. Soon after the play Sally takes her marbles and puts them into the basket and then she leaves the room. When Sally was out of the room, Anne took the marbles and placed them in her box and she also leaves the room. When Sally returns to the room, the experimenter asked the following question: "Where does Sally think her marble is?" After the response of the child, the second question was asked: "Where will Sally look for her marble?" and finally the last question was asked: "Where is the marble?"

\section{Task 2 (Smarty's Task)}

A chocolate box and a pencil were used as material. We put a pencil in the chocolate box. We showed the chocolate box to the child and asked two questions: "What is this?" and "What is in it?" The child was expected to answer "chocolate box" and "chocolate" for these questions.

In both the tasks, the responses of the child were scored as either true belief or false belief.

\section{Results}

The results of the present study revealed mixed responses in the preschoolers. It was found that 3- to 4-year-old children had more false beliefs for both the tasks when compared with 4- to 5-year-old children. Percentage of true belief and false belief across two tasks among 3- to 4- year-old preschoolers is represented in - Table 1. Percentage of true belief and false belief across the two tasks among 4 - to 5 -year-old preschoolers is represented in - Table 2. Overall percentage of true belief and false belief across the two tasks is represented in $\boldsymbol{- T a b l e} \mathbf{3}$.

Table 1 Percentage of true belief and false belief across two tasks among 3 to 4 years preschoolers

\begin{tabular}{|l|l|l|}
\hline Task & True belief & False belief \\
\hline Sally-Anne Task & $16 \%$ & $84 \%$ \\
\hline Smarty's Task & $22 \%$ & $78 \%$ \\
\hline
\end{tabular}

Table 2 Percentage of true belief and false belief across two tasks among 4 to 5 years preschoolers

\begin{tabular}{|l|l|l|}
\hline Task & True belief & False belief \\
\hline Sally-Anne Task & $84 \%$ & $16 \%$ \\
\hline Smarty's Task & $95 \%$ & $5 \%$ \\
\hline
\end{tabular}

Table 3 Overall percentage of true belief and false belief across two tasks

\begin{tabular}{|l|l|l|}
\hline Task & True belief & False belief \\
\hline Sally-Anne Task & $50 \%$ & $50 \%$ \\
\hline Smarty's Task & $58 \%$ & $42 \%$ \\
\hline
\end{tabular}




\section{Discussion}

Our results revealed a consistent increase in the ToM scores as age increased. By the age of 3, misconceptions belief is used by the children to conduct their deliberated and purposeful actions. At this period, children make predictions based on false beliefs to guide intentional actions on tasks. ${ }^{12}$ The 3-year-old child does not fully develop the ability to understand that other people's beliefs, desires, and knowledge may differ from their own. The 3-year-old thinks that everyone else knows what they know, sees what they see, and feels what they feel. ${ }^{12}$ This could be attributed to greater false belief response in our study. At the age of 4 to 5 years, the children will start to think or they will realize that people talk and act on the basis of the way they think what the world is, even when their thoughts do not reflect the real situation. These children know that people may think different things and they understand that sometimes a person may believe something that is not true but, in that case, what the person does or says is based on the false belief. ${ }^{13}$ By 4 years of age, most children can attribute mistaken beliefs to themselves and to others; thus, they begin to show new forms of social interaction such as tricks, jokes, cunning, and lying. In the play around the period of preschool age, the children will promote or shift their social partners, and they will also change their adult caregiver to friends, peer group, siblings, and cousins of the same age, i.e., they are not relying or requiring the conversational support of parents and adults. Most of the children like to interact and maintain connected conversations with their peer group, who also have the same shared interests, ideas, thoughts, humor, etc. ${ }^{14}$

\section{Conclusion}

It can be concluded that the ToM abilities improve in typically developing children. Future studies are required to explore the higher levels of embedding of ToM, and also to incorporate it in the clinical population. The results also stress on the need to develop a social competency scale suitable for Indian population.

\section{Conflict of Interest}

None declared.

\section{Acknowledgment}

The authors express sincere gratitude to The Director, Nitte Institute of Speech and Hearing and The Principal, Nitte Institute of Speech and Hearing for their constant support and encouragement. The authors also extend gratitude to the interns and the participants for their support.

\section{References}

1 Premack D, Woodruff G. Does the chimpanzee have a theory of mind? Behav Brain Sci 1978;1(4):515-526

2 Wimmer H, Perner J. Beliefs about beliefs: representation and constraining function of wrong beliefs in young children's understanding of deception. Cognition 1983;13(1):103-128

3 Baron-Cohen S, Leslie AM, Frith U. Does the autistic child have a "theory of mind"? Cognition 1985;21(1):37-46

4 Dunn J. Children as psychologists: The later correlates of individual differences in understanding of emotions and other minds. Cogn Emotion 1995;9(2-3):187-201

5 Cutting AL, Dunn J. The cost of understanding other people: social cognition predicts young children's sensitivity to criticism. J Child Psychol Psychiatry 2002;43(7):849-860

6 Dunn J, Brown J, Slomkowski C, Tesla C, Youngblade L. Young children's understanding of other people's feelings and beliefs: individual differences and their antecedents. Child Dev 1991;62(6):1352-1366

7 Hughes C, Dunn J. "Pretend you didn't know”: Preschoolers' talk about mental states in pretend play. Cogn Dev 1997;12(4): 477-497

8 Astington JW, Barriault T. Children's theory of mind: how young children come to understand that people have thoughts and feelings. Infants Young Child 2001;13(3):1-2

9 Flavell JH. Cognitive development: children's knowledge about the mind. Annu Rev Psychol 1999;50(1):21-45

10 Zahn-Waxler C, Radke-Yarrow M, Wagner E, Chapman M. Development of concern for others. Dev Psychol 1992; 28(1):126

11 Harris PL. The child's understanding of emotion: developmental change and the family environment. J Child Psychol Psychiatry 1994;35(1):3-28

12 Lee IR. The theory of mind: has your child developed it yet? 2017. Available at: https://wehavekids.com. Accessed April 27, 2019

13 Astington JW, Edward MJ. The development of theory of mind in early childhood. In: Tremblay RE, Boivin M, Peters $\mathrm{RDeV}$, eds. Zelazo PD, topic ed. Encyclopedia on Early Childhood Development [online]. Available at: http://www. child-encyclopedia.com/social-cognition/according-experts/ development-theory-mind-early-childhood. Accessed April 27, 2019

14 Dunn J. Changing Minds and Changing Relationships. Children's Early Understanding of Mind: origins and Development. Hillsdale, NJ: Lawrence Erlbaum Associates, Inc. 1994:297-310 